\title{
Reliability Modeling of Cloud-RAID-6 Storage System
}

\author{
Qisi Liu and Liudong Xing
}

\begin{abstract}
Cloud storage is a crucial component of cloud computing, where cloud storage providers supply a large amount of on-demand data storage so that users can save and retrieve their data anytime anywhere in the cloud. Any service interruption or failure could have negative and serious impact on the reputation of cloud service providers. Therefore, it is significant to model and design reliability for cloud storage systems. The RAID (Redundant Array of Independent Disks) technology is one of the most effective ways to achieve data reliability. There are several standard RAID levels with different fault tolerance techniques. In this paper, we model and evaluate reliability of RAID-6 configuration implemented in the cloud infrastructure (referred to as cloud-RAID-6). A hybrid analytical modeling method is proposed, which combines a Markov model for analyzing a single disk state probabilities and a multi-valued decision diagram model for analyzing the entire cloud-RAID-6 system state probabilities. The suggested method is applicable to identical and non-identical disks within the cloud-RAID-6. The proposed methodology is illustrated through examples.
\end{abstract}

Index Terms-Cloud-RAID-6, Markov model, multi-valued decision diagram, lattice structure, multi-state, reliability.

\section{INTRODUCTION}

According to the National Institute of Standards and Technology (NIST), cloud computing is described as a model that enables convenient, ubiquitous and on-demand network access to a shared pool of configurable computing resources [1]. Examples of the shared resources include servers, storages, services, applications, software, and etc. Providers can rapidly provide various resources in the form of services with the minimal management effort [1]. Based on their own needs, users can reuse or combine services available from the cloud anytime and anywhere, forming a loosely-coupled network application program. As a major revolution in the information industry, cloud computing is becoming an important and influential aspect of our way of life, and it has attracted considerable attentions from both academic and industrial communities.

It is a critical challenge to guarantee the reliability of cloud computing systems due to the dynamic environment [2], [3]. Particularly, resource allocation is typically done dynamically based on performance predictions in the cloud [4], [5]. For instance, resource allocation was done based on estimation of future loads on servers or Quality of Service (QoS) offered by a specific application [4], [5]. Due to factors such as wide area networks, large-scale service sharing, heterogeneous hardware and software components, and interactions among these components, traditional network

Manuscript received June 20, 2015; revised November 11, 2015

The authors are with the Department of Electrical and Computer Engineering, University of Massachusetts Dartmouth, MA 02747 USA (e-mail: qliu1@umassd.edu, lxing@umassd.edu). reliability models are not directly applicable to analyzing reliability of cloud computing systems [3].

In particular, cloud storage is a crucial component of cloud computing, where the storage providers (as a third party) supply a large amount of on-demand data storage to users [6]. Users are invisible to the complex underlying management with data migrating into cloud storage servers, and they expect to be able to access their data anytime anywhere. Therefore any service failure or interruption could have serious and negative impact on the reputation of cloud service providers. A number of major breakdowns of cloud computing has been reported in the last several years. For example, eight hours of unexpected outage of Simple Storage Service happened on the Amazon Cloud storage platform (Amazon S3), affecting numerous users [7]. Occurrence of such failures in services reflects the significance of addressing reliability issues for cloud storage systems.

While data reliability has become a significant metric of QoS, cloud-RAIDs using different data redundancy techniques are being designed and implemented as an effective solution to achieve high data reliability and they have been provided by several cloud storage providers [8]. Though there exist works modeling reliability of traditional RAID systems (see, e.g., [9]), they made a limited assumption that all the disks are located in the same place and are identical. In the cloud-RAID, data are distributed across multiple cloud storage providers and thus disks providing the service can be located in different sites and be statistically non-identical. Therefore, the existing methodology for RAID is not applicable to the reliability analysis of cloud-RAID.

In this paper, we propose a new hybrid analytical modeling methodology that consider both physical and logical architecture of cloud-RAID storage systems. The methodology integrates a Markov model and a multi-valued decision diagram (MDD) model. The Markov model is used to consider physical characteristics of each single disk and further analyze different disk state probabilities (or probability of disk being in different performance levels). The MDD model is used to analyze reliability of the entire cloud-RAID system that involves multiple disks located at different sites of the cloud.

The remainder of the paper is organized as follows. Section II presents background of the MDD model and its lattice structure. Section III presents an illustrative example of cloud-RAID-6 system. Section IV presents the proposed methodology. Section V discusses reliability analysis results. Finally Section VI concludes the paper.

\section{PRELIMINARY ON MDD}

As extensions of traditional binary decision diagrams, MDDs are graph-based data structures for representing and manipulating multi-valued logical functions [10], [11]. An 
MDD can express a multi-valued logical function as a directed acyclic graph that is both compact and canonical.

Let $f:\{0,1,2,3, \ldots, M\}^{w} \rightarrow\{0,1\}$ be a multi-valued logic function on a set of $(M+1)$-valued variables $\boldsymbol{x}=$ $\left\{x_{1}, x_{2}, \ldots, x_{w}\right\}$. Equation (1) givesthe decomposition of function $f$ with regard to variable $x_{i} \in \boldsymbol{x}$.

$$
f=\left(x_{i}=0\right) f_{x i=0}+\cdots+\left(x_{i}=M\right) f_{x i=M} .
$$

Equation (2) give the concise case format [12] of (1).

$$
\begin{aligned}
f= & \operatorname{case}\left(x_{i}, f_{x=0}, f_{x i=1}, \ldots, f_{x i=M}\right) \\
& =\operatorname{case}\left(x_{i}, f_{0}, f_{1} \ldots, f_{M}\right) .
\end{aligned}
$$

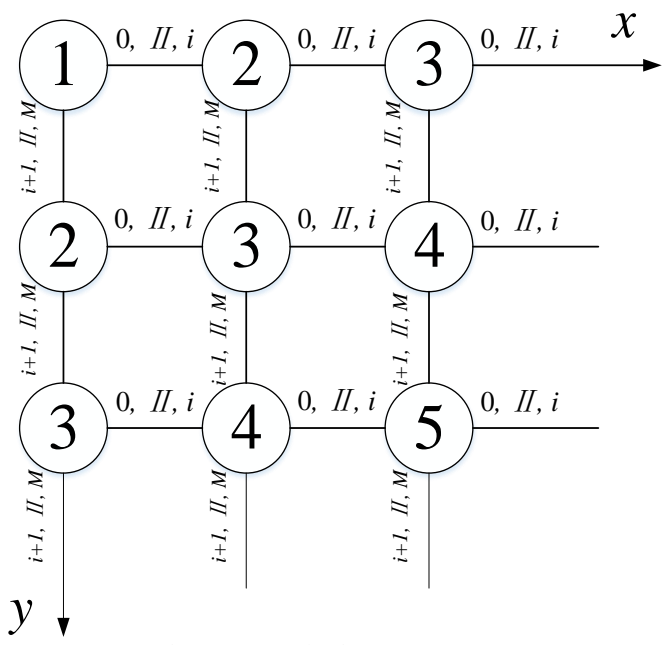

Fig. 1. MDD lattice structure.

An MDD is a rooted graph, consisting of one or more decision nodes (or non-sink nodes) and two terminal nodes (or sink nodes). The two terminal nodes are labeled ' 0 ' and ' 1 ', representing the modeled system not being or being in a particular system state, respectively.

Each non-sink node in an MDD encodes a case construct of (2). Specifically, each non-sink node is associated with a multi-valued variable $x_{i}$ and has $(M+1)$ outgoing edges. The $j$-th edge $(0 \leq j \leq M)$ is connected to a child node encoding the multi-valued function $f_{x i=j}$. During the MDD generation, if all the multi-valued variables are ordered, an ordered MDD (OMDD) can be obtained, where every root node to sink node path visits variables in an ascending order. Furthermore by applying two reduction rules of "merging isomorphic subtrees" and "deletion of useless nodes", a reduced OMDD (ROMDD) can be obtained [12]. Each path from root-to-sink node ' 1 ' represents a disjoint combination of component states that can lead to the system being in a particular state. Therefore, the probability of a system being in a particular state can be obtained by adding probabilities of all paths from root to sink node ' 1 ' of the corresponding system state MDD model.

In the case of modeling a multi-state system whose structure function depends on at least $K$ out of $N$ components being at state $i+1$ or above, a well-defined lattice structure of MDD model can be constructed. Fig. 1 illustrates the lattice structure of MDD model, which is an extension of the lattice structure of binary decision diagrams for binary state systems constructed in [13]. The MDD lattice structure is applied in Section IV to model reliability of the cloud-RAID-6 system.

\section{Cloud-RAID-6 STORAge SySTEM}

RAID technology is one of the most effective ways to achieve data reliability and protect the data from a disk failure. There exist several standard RAID levels, which utilize different fault tolerance methods such as striping, mirroring, and parity to store data onto multiple hard disk drives [14].

Among all the levels, RAID-6 is one of the levels that can provide protection for data from concurrent faults of any two disk drives by using two independent distributed parity blocks. In this paper, we study cloud-RAID-6, which is the RAID-6 configuration being implemented the cloud infrastructure where different disk drives can be provided by different storage system providers.

In cloud-RAID-6, the data is split into several columns with evenly distributed parity blocks and spread across multiple providers to tolerate possible failures. Just as using two disks for double checking in the traditional RAID-6, these parity blocks require two complete columns of storage space from any two of the multiple cloud storage providers for data redundancy. The storage space utilization is $(n-2) / n$ if there are $n$ cloud storage providers in the cloud-RAID-6 storage system.

Fig. 2 illustrates the basic architecture of an example cloud-RAID-6 storage system. The data is spilt into stripes saved in 5 columns (supported by 5 cloud storage providers) with $5 \boldsymbol{P}$ plus $5 \boldsymbol{Q}$ redundancy arrays evenly distributed. If any two of the cloud storage providers are not available, the whole storage system can still operate due to the data redundancy. The lost data are able to be retrieved using the parity blocks and remaining data stripes saved by other cloud storage providers, thus the entire storage system can be reconstructed.

Specifically, in the example cloud-RAID-6, the $\boldsymbol{P}$ parity stripe $A_{p}$ can be simply generated by applying exclusive $O R$ (XOR) operation to $A_{1}, A_{2}$, and $A_{3}$ :

$$
A_{p}=A_{1} X 0 R A_{2} X O R A_{3}
$$

The $\boldsymbol{Q}$ parity stripe $\mathrm{A}_{\mathrm{q}}$ is calculated using Reed-Solomon coding with the algebra of suitable Galois field.

$$
A_{q}=g^{0} A_{1} X 0 R g^{1} A_{2} X O R g^{2} A_{3}
$$

where $g$ is some generator of a certain Galois field.

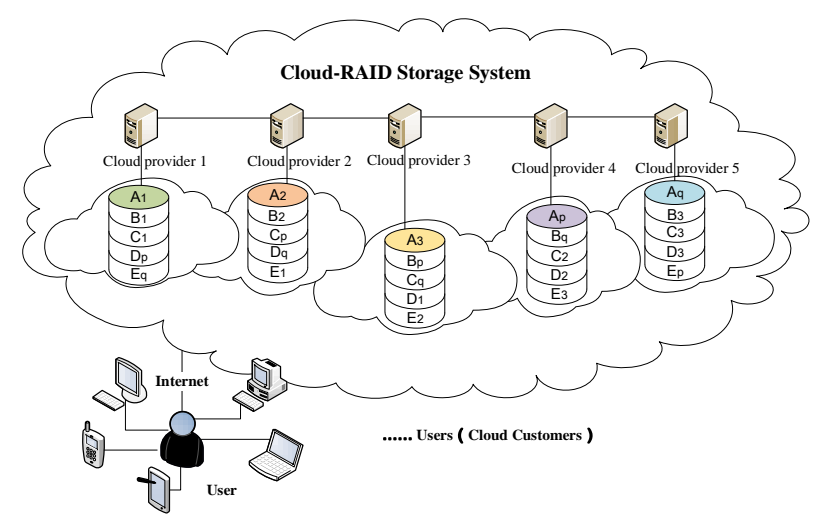

Fig. 2. Architecture of an example cloud-RAID-6 storage system.

For instance, if the service from the cloud storage provider 
1 fails, the unavailable data stripe $A_{1}$ is no longer accessible, but it can be recovered by XORing the parity stripe $A_{p}$ from cloud provider 4 and the remaining data stripes $A_{2}$ and $A_{3}$. If one parity stripe $A_{p}$ or $A_{q}$ is lost, we can just re-compute.

However, when two failures occur at the same time, for example, one data stripe like $A_{1}$ and $P$ parity stripe $A_{p}$ are lost at the same time, or one data $\mathrm{A}_{1}$ and one $\boldsymbol{Q}$ parity stripe $A_{q}$ are lost, or two data $A_{1}, A_{2}$ are lost, the data can be recovered from $\boldsymbol{P}$ and $\boldsymbol{Q}$ parity syndromes by using a more complicated method described in [15].

\section{Proposed Hybid Evaluation Method}

Based on the example configuration in the Fig. 2, we consider the basic architecture of the cloud-RAID- 6 in two levels:

1) The cloud-RAID components: storing space in columns provided by one cloud server considered as an independent virtual disk drive.

2) The cloud-RAID system: the storage system in cloud environment contributed by multiple cloud storage providers.

At level 1, we use a Markov process to model failure and recovery behavior of an individual disk with the assumption of exponential failure and restoration time. At level 2, we explore an MDD-based $K$-out-of- $N$ model for evaluating the reliability of cloud-RAID-6 that consists of multiple disks from different providers. In light of the proposed hierarchical approach, impacts of some important parameters like disk transition rate $\lambda$, recovery rate $\mu$ on the reliability of cloud-RAID-6 storage system are examined.

\section{A. Individual Disk State Probabilities}

Based on previous studies in [9] and [16], a disk drive in RAID can assume the following three states: G (good state), D (degraded state), or F (permanent failed state). Each disk has a self-recovery mechanism with recovery rate $\mu$ which is also called media repair or data retrieve as shown in Fig. 3.

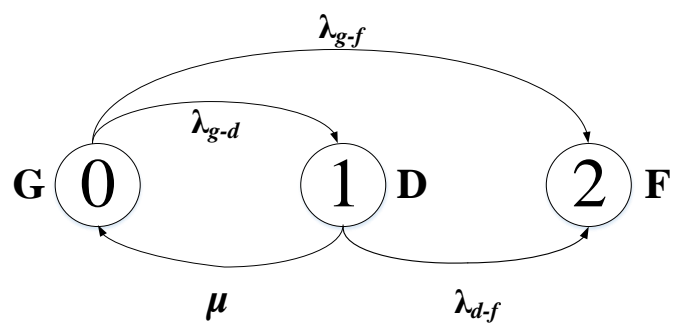

Fig. 3. Markov model for a single disk.

When a disk is in state $\mathbf{G}$ and $\mathbf{D}$, the disk is operating correctly. The disk in state $\mathbf{G}$ may enter state $\mathbf{D}$ with transition rate $\lambda_{g-d}$ or directly goes to state $\mathbf{F}$ with transition rate $\lambda_{g-f}$ owing to unrepaired faults or permanent data lost. A disk in state $\mathbf{D}$ may return to state $\mathbf{G}$ with recovery rate $\mu$ after successfully performing the related restoration process or may go to state $\mathbf{F}$ with transition rate $\lambda_{d-f}$ if the restoration fails.

Let $P_{0}(t), P_{1}(t), P_{2}(t)$ represent probabilities that the disk is in state $\mathbf{G}, \mathbf{D}$ and $\mathbf{F}$ at time $t$, respectively. Based on the Markov model in Fig. 3, the following equations are obtained.

$$
\begin{gathered}
\dot{P}_{0}(t)=-\left(\lambda_{g-d}+\lambda_{g-f}\right) P_{0}(t)+\mu P_{1}(t) \\
\dot{P}_{1}(t)=\lambda_{g-d} P_{0}(t)-\left(\mu+\lambda_{d-f}\right) P_{1}(t) \\
\dot{P}_{2}(t)=\lambda_{g-f} P_{o}(t)+\lambda_{d-f} P_{1}(t)
\end{gathered}
$$

Applying Laplace transform with the assumption of initial probability of the disk being $P_{0}(0)=1$, the probabilities of the disk being in the good state $\mathbf{G}$ and degradation state $\mathbf{D}$ are

$$
\begin{gathered}
P_{0}(t)=\frac{\mu+\lambda_{d-f}-c_{1}}{c_{2}-c_{1}} e^{-c_{1} t}+\frac{\mu+\lambda_{d-f}-c_{2}}{c_{1}-c_{2}} e^{-c_{2} t} \\
P_{1}(t)=\frac{\lambda_{g-d}}{c_{2}-c_{1}} e^{-c_{1} t}+\frac{\lambda_{g-d}}{c_{1}-c_{2}} e^{-c_{2} t}
\end{gathered}
$$

where

$$
\begin{aligned}
& c_{1}=0.5\left(\operatorname{Sum}+\sqrt{\operatorname{Sum}^{2}-4\left(\lambda_{g-d} \lambda_{d-f}+\lambda_{g-f} \lambda_{d-f}+\lambda_{g-f} \mu\right)}\right) \\
& c_{2}=0.5\left(\operatorname{Sum}+\sqrt{\operatorname{Sum}^{2}-4\left(\lambda_{g-d} \lambda_{d-f}+\lambda_{g-f} \lambda_{d-f}+\lambda_{g-f} \mu\right)}\right) \\
& S u m=\mu+\lambda_{g-d}+\lambda_{g-f}+\lambda_{d-f} .
\end{aligned}
$$

The probability of the disk being in the permanent failure state is thus

$$
\begin{gathered}
P_{2}(t)=1-P_{0}(t)-P_{1}(t) \\
= \\
1-\frac{\mu+\lambda_{g-d}+\lambda_{d-f}-c_{1}}{c_{2}-c_{1}} e^{-c_{1} t}-\frac{\mu+\lambda_{g-d}+\lambda_{d-f}-c_{2}}{c_{1}-c_{2}} e^{-c_{2} t}
\end{gathered}
$$

Equation (8) also gives unreliability of the disk.

\section{B. Cloud-RAID-6 System State Probabilities}

The ground of this approach is to develop an analytical model for reliability evaluation of an overall cloud-RAID storage system. As illustrated in Fig. 4, each individual disk can be defined as a three-state component in this paper, modeled by a non-sink node in the MDD model. In particular, the disk $k(k=1,2,3,4,5)$ that can be in states $\mathbf{G}, \mathbf{D}$, and $\mathbf{F}$ is displayed by a non-sink node with three outgoing edges, associated with probabilities $P_{0 k}, P_{1 k}, P_{2 k}$, respectively.

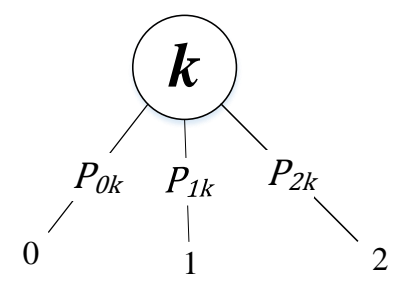

Fig. 4. MDD for disk $k(k=1,2,3,4,5)$.

The entire cloud-RAID 6 system also has three states: good, degraded, and failed associated with probabilities $P_{s=0}(t), P_{s=1}(t)$ and $P_{s=2}(t)$ respectively.

The example cloud-RAID-6 system is considered being in a good state when at least 3 disks are in state $\mathbf{G}$, modeled using 3-out-of-5 MDD lattice structure in Fig. 5. The two sink nodes ' 1 ' and ' 0 ' means the system is or is not in the 
good state.

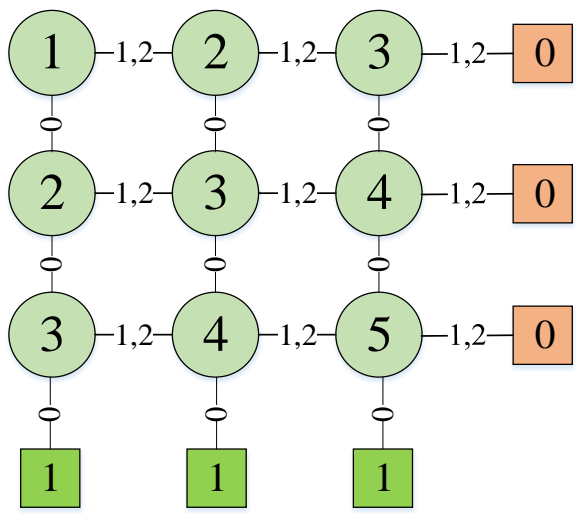

Fig. 5. MDD of system being in good state 0 .

The probability of the system being in the good state $P_{S=0}(t)$ is given as the sum of probabilities of all paths from the root node 1 to sink node ' 1 ' in the MDD of Fig. 5.

$$
\begin{aligned}
& P_{s=0}=P_{01} P_{02} P_{03}+P_{01} P_{02}\left(1-P_{03}\right) P_{04} \\
& +P_{01} P_{02}\left(1-P_{03}\right)\left(1-P_{04}\right) P_{05} \\
& \quad+P_{01}\left(1-P_{02}\right) P_{03} P_{04} \\
& \quad+P_{01}\left(1-P_{02}\right) P_{03}\left(1-P_{04}\right) P_{05} \\
& \quad+P_{01}\left(1-P_{02}\right)\left(1-P_{03}\right) P_{04} P_{05} \\
& \quad+\left(1-P_{01}\right) P_{02} P_{03}\left(1-P_{04}\right) P_{05} \\
& +\left(1-P_{01}\right)\left(1-P_{02}\right) P_{03} P_{04} P_{05}(9)
\end{aligned}
$$

Similarly, the entire cloud-RAID-6 system is considered being in a failed state when 3 disks or more are in state $\mathbf{F}$ modeled using 3-out-of-5 MDD lattice structure in Fig. 6. Sink node ' 1 ' in Fig. 6 means the system is in the failed state; sink node ' 0 ' means the system is not in the failed state.

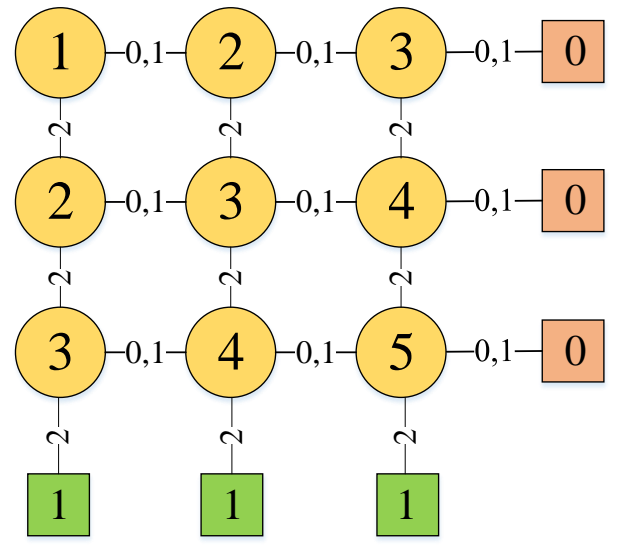

Fig. 6. MDD of system being in failed state 2 .

Evaluating the MDD in Fig. 6, the probability of the system being in the failed state $P_{s=2}(t)$ is

$$
\begin{aligned}
P_{s=2} & =P_{21} P_{22} P_{23}+P_{21} P_{22}\left(1-P_{23}\right) P_{24} \\
& +P_{21} P_{22}\left(1-P_{23}\right)\left(1-P_{24}\right) P_{25} \\
& +P_{21}\left(1-P_{22}\right) P_{23} P_{24} \\
& +P_{21}\left(1-P_{22}\right) P_{23}\left(1-P_{24}\right) P_{25} \\
& +P_{21}\left(1-P_{22}\right)\left(1-P_{23}\right) P_{24} P_{25} \\
& +\left(1-P_{21}\right) P_{22} P_{23}\left(1-P_{24}\right) P_{25} \\
& +\left(1-P_{21}\right)\left(1-P_{22}\right) P_{23} P_{24} P_{25}(10)
\end{aligned}
$$

Any state in-between the good state and failed state is considered as a degraded state. The probability that the system is in the degraded state can thus be simply obtained as

$$
P_{s=1}(t)=1-P_{s=2}(t)-P_{s=0}(t)
$$

\section{ANALYSIS RESULTS}

In this section numerical results are presented for the example cloud-RAID-6 system.

\section{A. Individual Disk State Probabilities}

Table I shows transition rates $\lambda_{g-d}, \lambda_{g-f}, \lambda_{d-f}$ (numbers of faults per hour) and recovery rate $\mu$ for the five disks of the example cloud-RAID-6 system.

TABLE I: TRANSITION RATES AND RECOVERY RATE FOR EACH DISK

\begin{tabular}{ccccc}
\hline \hline Disk $k$ & $\mu$ & $\lambda_{g-d}$ & $\lambda_{g-f}$ & $\lambda_{d-f}$ \\
\hline 1 & 0.01 & 0.00015 & 0.00002 & 0.0002 \\
2 & 0.05 & 0.0002 & 0.000019 & 0.00019 \\
3 & 0.16 & 0.0001 & 0.000025 & 0.00026 \\
4 & 0.24 & 0.0003 & 0.000013 & 0.0004 \\
5 & 0.13 & 0.00025 & 0.00001 & 0.00032 \\
\hline \hline
\end{tabular}

Tables II, III, IV show probabilities of individual disks and the entire example cloud-RAID-6 system being in the good, degraded, and failed states for different mission times (in hours), respectively. The state probabilities for each disk $P_{k 0}, P_{k 1}$ and $P_{k 2}$ are calculated by (6), (7) and (8), respectively.

The state probabilities of the entire cloud-RAID- 6 system $P_{S=0}, P_{S=1}$ and $P_{S=2}$ are computed by (9), (10) and (11), respectively.

\section{B. Individual Disk State Probabilities}

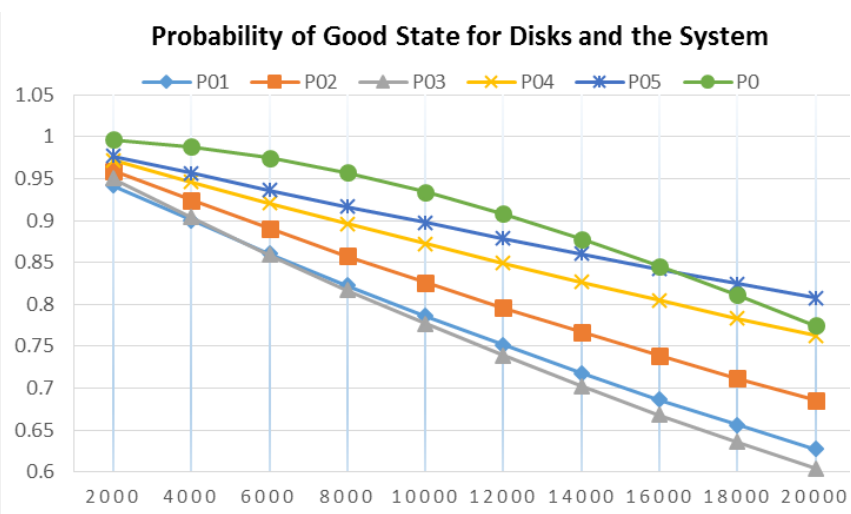

Fig. 7. Probability of good state for each disk and the system.

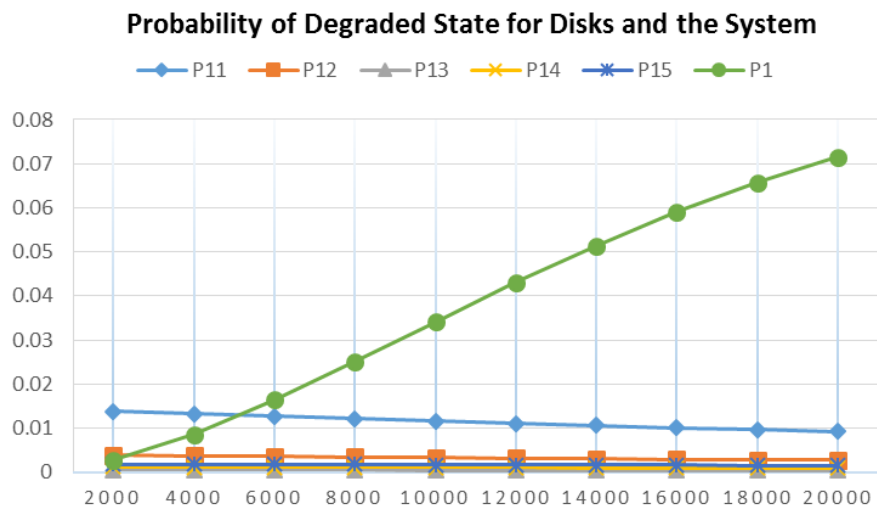

Fig. 8. Probability of degraded state for each disk and the system. 


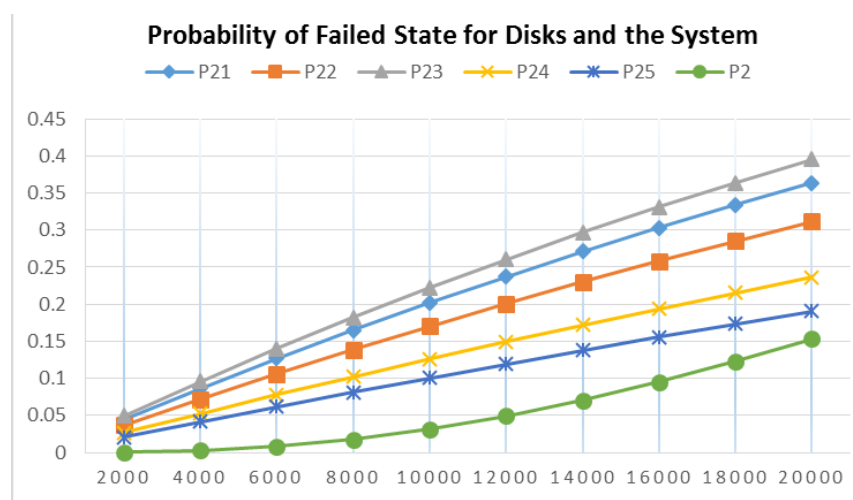

Fig. 9. Probability of failed state for each disk and the system.
Probability of Each State for the Cloud-RAID Storage System

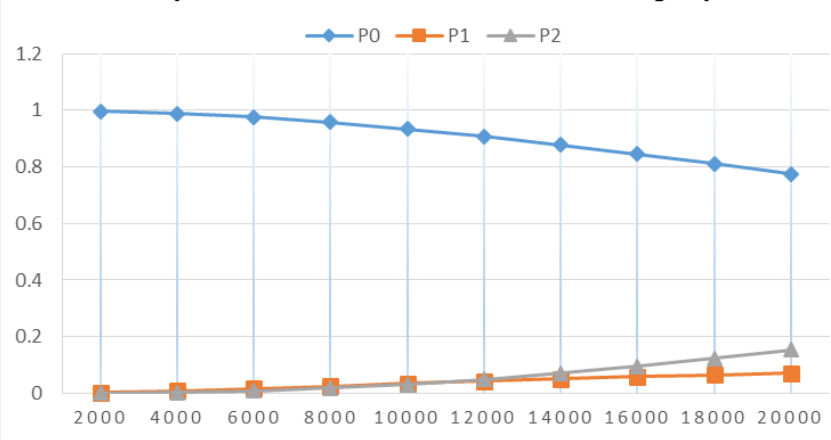

Fig. 10. Probability of good, degraded, failed states for the system.

TABLE II: PROBABILITY OF GOOD STATE FOR INDIVIDUAL DISKS AND THE SYSTEM

\begin{tabular}{|c|c|c|c|c|c|c|c|c|c|c|}
\hline 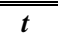 & 2000 & 44000 & 6000 & 8000 & "10000 & "12000 & 14000 & 16000 & "18000 & 20000 \\
\hline$P_{01}$ & 0.942135 & 0.900473 & 0.860653 & 0.822594 & 0.786217 & 0.751450 & 0.718220 & 0.686459 & 0.656103 & 0.627090 \\
\hline$P_{02}$ & 0.959512 & 0.924320 & 0.890420 & 0.857762 & 0.826303 & 0.795997 & 0.766803 & 0.738679 & 0.711587 & 0.685489 \\
\hline$P_{03}$ & 0.950358 & 0.903744 & 0.859416 & 0.817262 & 0.777176 & 0.739056 & 0.702806 & 0.668333 & 0.635552 & 0.604379 \\
\hline$P_{04}$ & 0.972184 & 0.946320 & 0.921144 & 0.896637 & 0.872782 & 0.849563 & 0.826960 & 0.804960 & 0.783544 & 0.762698 \\
\hline$P_{05}$ & 0.977165 & 0.956680 & 0.936624 & 0.916988 & 0.897764 & 0.878943 & 0.860516 & 0.842476 & 0.824814 & 0.807523 \\
\hline$P_{s=0}$ & 0.996836 & 0.988632 & 0.975313 & 0.957119 & 0.934482 & 0.907950 & 0.878127 & 0.845631 & 0.811066 & 0.775003 \\
\hline
\end{tabular}

TABLE III: PRobabILITY OF DEGRADED STATE FOR INDIVIDUAL DISKS AND THE SYSTEM

\begin{tabular}{|c|c|c|c|c|c|c|c|c|c|c|}
\hline$t$ & 2000 & 4000 & 6000 & 8000 & 10000 & 12000 & 14000 & 16000 & 18000 & 20000 \\
\hline$P_{11}$ & 0.013886 & 0.013272 & 0.012685 & 0.012124 & 0.011588 & 0.011075 & 0.010586 & 0.010117 & 0.009670 & 0.009242 \\
\hline$P_{12}$ & 0.003825 & 0.003685 & 0.003550 & 0.003419 & 0.003294 & 0.003173 & 0.003057 & 0.002945 & 0.002837 & 0.002733 \\
\hline$P_{13}$ & 0.000593 & 0.000564 & 0.000536 & 0.000510 & 0.000485 & 0.000461 & 0.000439 & 0.000417 & 0.000397 & 0.000377 \\
\hline$P_{14}$ & 0.001213 & 0.001181 & 0.001150 & 0.001119 & 0.001089 & 0.001060 & 0.001032 & 0.001005 & 0.000978 & 0.000952 \\
\hline$P_{15}$ & 0.001875 & 0.001835 & 0.001797 & 0.001759 & 0.001722 & 0.001686 & 0.001651 & 0.001616 & 0.001582 & 0.001549 \\
\hline$P_{s=1}$ & 0.002786 & 0.008643 & 0.016386 & 0.025114 & 0.034157 & 0.043031 & 0.051395 & 0.059021 & 0.065770 & 0.071568 \\
\hline
\end{tabular}

TABLE IV: PROBABILITY OF FAILED STATE FOR INDIVIDUAL DISKS AND THE SYSTEM

\begin{tabular}{|c|c|c|c|c|c|c|c|c|c|c|}
\hline$t$ & 2000 & 4000 & 6000 & 8000 & 10000 & 12000 & 14000 & 16000 & 18000 & 20000 \\
\hline$P_{21}$ & 0.043979 & 0.086256 & 0.126663 & 0.165283 & 0.202195 & 0.237475 & 0.271195 & 0.303423 & 0.334227 & 0.363668 \\
\hline$P_{22}$ & 0.036663 & 0.071995 & 0.106031 & 0.138818 & 0.170403 & 0.200830 & 0.230141 & 0.258376 & 0.285576 & 0.311779 \\
\hline$P_{23}$ & 0.049049 & 0.095692 & 0.140048 & 0.182228 & 0.222339 & 0.260483 & 0.296756 & 0.331249 & 0.364051 & 0.395244 \\
\hline$P_{24}$ & 0.026602 & 0.052499 & 0.077707 & 0.102244 & 0.126128 & 0.149377 & 0.172007 & 0.194036 & 0.215478 & 0.236350 \\
\hline$P_{25}$ & 0.020960 & 0.041485 & 0.061579 & 0.081253 & 0.100514 & 0.119371 & 0.137833 & 0.155907 & 0.173603 & 0.190928 \\
\hline$P_{S=2}$ & 0.000377 & 0.002725 & 0.008301 & 0.017767 & 0.031360 & 0.049019 & 0.070478 & 0.095348 & 0.123164 & 0.153430 \\
\hline
\end{tabular}

Fig. 7, Fig. 8, and Fig. 9 respectively show the trend for the probability of being at the good state, degradation state and failure state over the period from 2,000 to 20,000hours for each disk $P_{k}(k=1,2,3,4,5)$ and the entire system $P_{s}$. Fig. 10 summarizes the state probabilities of the entire system.

Obviously in Fig. 7, the system good state probability $P_{s=0}$ stays at a higher level until 16,000 hours while all probabilities for individual disks and the system decrease gradually with the time increasing. With appropriate implementation of the RAID-6 technology into cloud infrastructure, the cloud-RAID can significantly improve the performance of cloud storage system rather than using a single disk from one cloud provider. After 16,000 hours, $P_{s=0}$ drops to around 0.8 , worse than the most reliable disk but still perform better than other disks. However this phenomenon shows that the advantage of the redundant architecture of cloud-RAID is related to time.

As shown in Fig. 8, there is a big gap between the probability of degraded state for each disk and for the entire system. The probability of the system being at the degraded state $P_{s=1}$ increases sharply, peaking at about 0.07 during the considered mission time while most of single disk degraded state probabilities $P_{k 1}$ decline slowly below 0.01 . From Fig. 8 , we also observe that the probability of disk 1 being at the degraded state $P_{11}$ is a little bit higher than the degraded state probabilities of other disks. This is because the recovery rate $\mu$ for disk1 (0.01) is less than other disks, which reflects the impact of recovery rate $\mu$ on the performance of each disk.

\section{CONCLUSION}

In this paper, we model a fault-tolerant cloud-RAID-6 storage system that distributes data and double parity to multiple non-identical disks from different cloud storage providers. A hybrid analytical modeling methodology is proposed. At the lower level, a Markov model is used for considering physical failure and recovery behaviors of a single disk and further for analyzing the probabilities of the disk being at three different performance levels or states. At the higher level, an efficient MDD model is utilized for modeling the fault tolerant behavior of multiple disks and further analyzing the probabilities of the entire cloud-RAID-6 system being at three different states. The failure state probability gives the unreliability of the entire cloud-RAID-6 system. The benefit and feasibility of the cloud-RAID-6 storage model is verified by comparing the state probabilities of the example cloud-RAID-6 system with those of its individual disks.

In this work we have assumed perfectly reliable links in the 
reliability modeling of cloud-RAID-6 systems. We will relax this assumption in our future work. We will also investigate other levels of RAID architecture for reliability modeling and evaluation of cloud storage systems.

\section{REFERENCES}

[1] T. Erl, R. Puttini, and Z. Mahmood, Cloud Computing Concepts, Technology \& Architecture, 1st ed., The Prentice Hall Service Technology Series, Prentice Hall, 2013.

[2] Y. Zhang, Z. Zheng, and M. R. Lyu, "BFT Cloud: A byzantine fault tolerance framework for voluntary-resource cloud computing," in Proc. IEEE International Conference on Cloud Computing, Washington. DC, 2011, pp. 444-451.

[3] J. Deng, S. C.-H. Huang, Y. S. Han, and J. H. Deng, "Fault tolerant and reliable computation in cloud computing," in Proc. IEEE Globecom Workshops, FL: Miami, December 2010, pp. 1601-1605.

[4] S. Pacheco-Sanchez, G. Casale, B. Scotney, S. McClean, G. Parr, and S Dawson, "Markovian workload characterization for QoS prediction in the cloud," in Proc. IEEE International Conference on Cloud Computing, Washington. DC, July 2011, pp. 147-154.

[5] K. V. Vishwanath and N. Nagappan, "Characterizing cloud Computing hardware reliability," in Proc. the First ACM Symposium on Cloud Computing, 2010, pp. 193-204.

[6] J. Strickland. (March 2015). How cloud storage works. [Online]. Available:

http://computer.howstuffworks.com/cloud-computing/cloudstorage.ht $\mathrm{ml}$

[7] G. Robinson, A. Narin, and C. Elleman. "Using Amazon web services for disaster recovery," presented at the Amazon Web Services, 2013.

[8] F. Bausch. (March 2015). Cloud-RAID concept. [Online]. Available: http://blog.fbausch.de/cloudraid-3-concept/

[9] T. Jin, L. Xing, and Y. Yu, "A hierarchical Markov reliability model for data storage systems with media self-recovery," International Journal of Reliability, Quality and Safety Engineering, vol. 18, no. 1, pp. 25-41, 2011.

[10] T. Kam, T. Villa, R. Brayton, A. Sangiovanni-Vincentelli, Synthesis of Finite State Machines: Functional Optimization, Kluwer Academic Publishers, MA: Boston, 1997.

[11] A. Shrestha, L. Xing, and Y. Dai, "Decision diagram-based methods, and complexity analysis for multistate systems," IEEE Trans. on Reliability, vol. 59, no. 1, pp. 145-161, March 2010.

[12] L. Xing and Y. Dai, "A new decision diagram based method for efficient analysis on multi-state systems," IEEE Trans. on Dependable and Secure Computing, vol. 6, No. 3, pp. 161-174, July-Sept. 2009.

[13] Y. Mo, L. Xing, S. V. Amari, and J. B. Dugan, "Efficient analysis of multi-state k-out-of-n systems," Reliability Engineering \& System Safety, vol. 133, pp. 95-105, 2015.
[14] D. A. Patterson, P. Chen, G. Gibson, and R. H. Katz, "Introduction to redundant arrays of inexpensive disks (RAID)," presented at the Thirty-Fourth IEEE Computer Society International Conference: Intellectual Leverage, Digest of Papers, pp. 112-117, San Francisco, CA, USA, 1989

[15] H. P. Anvin, The Mathematics of RAID-6, 2007.

[16] H. Pham, A. Suprasad, and R. B. Misra, "Reliability analysis of k-out-of-n systems with partially reparable multi-state components," Microelectronics Reliability, vol. 36, no. 10, pp. 1407-1415, 1996.

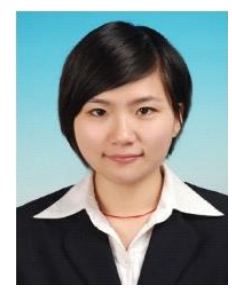

Qisi Liu was born in China, 1990. She earned the B.E. degree in mechatronic engineering from Donghua University, China in 2012, and the M.S. degree in computer engineering from the University of Massachusetts Dartmouth in January 2016.

She is currently a Ph.D. student in the Department of Electrical and Computer Engineering, University of Massachusetts Dartmouth, USA. Her research interest is in the field of reliability and risk modeling of complex systems such as cloud computing and financial systems.

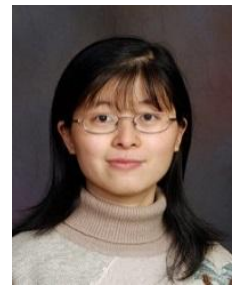

Liudong Xing received the B.E. degree in computer science from Zhengzhou University, China in 1996 and the M.S. and Ph.D. degrees in electrical engineering from the University of Virginia in 2000 and 2002, respectively.

She is currently a professor with the Department of Electrical and Computer Engineering, University of Massachusetts (UMass) Dartmouth, USA. She is an author or co-author of over 200 technical papers and a book entitled "Binary Decision Diagrams and Extensions for System Reliability Analysis". Her research focuses on reliability modeling and analysis of complex systems and networks.

Prof. Xing is an associate editor for International Journal of Systems Science (IJSS), IJSS: Operations \& Logistics, and Journal of Computational Engineering. She is also an assistant editor-in-chief for International Journal of Performability Engineering, and an editorial board member for Reliability Engineering \& System Safety. She is the recipient of UMass Dartmouth Leo M. Sullivan Teacher of the Year Award (2014), Scholar of the Year Award (2010), and Outstanding Women Award (2011). She is also the recipient of the 2007 IEEE Region 1 Technological Innovation (Academic) Award and the co-recipient of the Best Paper Award at the 2009 IEEE International Conference on Networking, Architecture, and Storage. She is a senior member of IEEE. 